if he would outline the differences, instead of saying they are different, which everybody knows. The same trend of thought is noticeable in his attitude toward paresis. Alcohol does not eause true paresis; alcoholic pseudoparesis is another thing. This is true, but, continued Dr. Jelliffe, what we want to know, and what a number of students have been trying to tell us is, how are we, from a clinical point of view, going to decide in the small number of cases where a differential is necessary? The paretic syndrome is so closely simulated in chronic alcobolism that the autopsy findings alone will clear up the diff. culties-in a small number of cases it is true; but advance in diagnostic skill means the cracking of the hard nuts and not the finding of self-evident answers. Dr. Gordon is fortunate in being able to diagnose all of his cases of chronic alcoholism from true paresis. Kraepelin, Bonhoffer, Cramer, Ziehen, Bianchi and most psychiaters have said that in a small num. ber of cases the autopsy alone will decide the question. Such has been Dr. Jelliffe's experience. Of late years the finding of the lymphocytes in the cerebrospinal fluid, the specific antibodies of Wassermann and Plant, etc., have aided in the diagnosis, but everywhere syphilis is a complicating factor, and cerebrospinal syphilis itself is not paresis. The problem which Dr. Gordon has stated is correct: paresis is not alcoholism; no one of standing says it is; but more clear symptom pictures are needed to guide us in the unraveling of the doubtful cases. These, it does not seem, Dr. Gordon has contributed. Another point of considerable forensic interest, said Dr. Jelliffe, is the effects of alcohol on superior degenerates or psychopathic inferiors, as Kraepelin terms them. Here the study of the development of paranoid complexes is highly demanded from the medicolegal side. That such individuals are more susceptible to alcohol is well known, and the tendencies to de. lusional developments are in need of more precise knowledge. Dr. Gordon said nothing of Korsakow's phenomenon, its dis. cussion with pseudoparesis, Dr. Jellifie thought, would seem to be necessary.

Dr. F. X. Dercum, Philadelphia, said that in his lectures on mental diseases he has adopted a point of view almost identical with that of Dr. Gordon. He has always held that the manifestations of alcohol were purely those of toxicity and must be distinguished from those of the purely degenerative psychopathies.

The differential diagnosis between paresis and alcoholism must be made by the physical signs. If there be a typical paretic speech, unequal and Argyll-Robertson pupils, tremor of lips and tongue, facial twitchings, etc., the case should be regarded as one of paresis. In making the differentiation, we should, of course, bear in mind the fact that alcoholism is not infrequently a complication of paresis, the paretic, now and then, drinking to excess. The same thing holds true with regard to paranoia. Alcohol bears no direct relations to paranoia. Occasionally, however, paranoiacs become alcoholics and the alcohol in turn hastens the mental degeneration and accentuates the paranoid symptoms, but alcohol does not, of itself, induce paranoia.

Dr. Alfred Gordon, Philadelphia, answering Dr. Jelliffe's question about the course of Korsakoff's psychoses, said he did not make a study of these psychoses in his cases, although he observed in some cases a typical course of these psychoses. They were practically all eases of acute alcoholism; in very few cases were there so-called fabrications. There was nothing especial about the confusional state or the delirious state. As to the effect of alcohol on certain degenerations, Dr. Gordon said that he had undertaken his study with one object in view, viz., to determine the effect of alcohol on the patients and to make an endeavor to differentiate between alcoholic mental states and other psychoses. Dr. Gordon always avoids using the term paranoia, or melancholia, or mania in association with other possible toxic conditions. He urged that every man should avoid the terms paranoia complex and alcoholic paranoia, etc. It is extremely confusing. Paranoia is a distinct malady, which has a special symptomatology, a special onset, and a special determination. We have no right to speak of secondary paranoia, alcoholic paranoia, if we want to be scientifically accurate. It is true, he said, that in some cases there is a difficulty in differentiating the alcoholic from the other paranoias, but if sufficient time is spent in the study of cases of this character the difficulty may be overcome.

Dr. Gordon's study of over 400 cases leads him to conclude that, while there are cases in which it is difficult to make a sharp, clear-cut diagnosis between the two conditions, in the majority of cases, the diagnosis is comparatively easy. In conclusion, Dr. Gordon emphasized that the paranoiac and the paretic may drink, but the alcohol has nothing to do (as a cause) with the mental symptoms or with the previous condition, except that it aggravates the pre-existing mental disturbances.

\section{THE DISADVANTAGES OF LOW PROTEIDS IN INFANT FEEDING.*}

\section{ALFRED HAND, JR., M.D. PHILADELPHIA.}

It seems hardly necessary at the present time to bring up the subject of infant feeding, especially as there is nothing new in this communication, but it is only for the purpose of emphasizing a certain point which has, perhaps, not received sufficient attention.

The first statement usually made with regard to bottle feeding is that cow's milk, the best available substitute for mother's milk, is too high in its proteid content for the infant stomach to digest. In avoiding this feature, it seems as though there had been for a time a tendency to go to the other extreme and feed infants with mixtures that were too dilute.

This phase of infant feeding was impressed on me some years ago when I first began to direct the feeding of infants. In the considerable number of children passing through my dispensaries there were soon recognized four types of infants; for the saying that "a man is what he eats" is nowhere better illustrated than in infancy. These types were as follows:

1. The Normal Type--The healthy, breast-fed infant, seen relatively infrequently, coming with some minor disorder from which recovery was prompt, and presenting the curved lines of beauty in the plump form, a pink glow of health, and a fat face bearing the heavy expression of contentment.

2. The Condensed Milk Type.-These infants appear fat, but are usually under weight, and sometimes thin; they are always rachitic and anemic, having a characteristic, bluish-white appearance like a delicate piece of china.

3. The Malnutrition Type.-It is perhaps too harsh to term this group the proprietary-food type, for there are many causes of marasmus, but the usual history was that one or more (several as a rule) of the proprietary foods had been used. This type comprised the greatest number, coming mainly for acute ileocolitis or simple indigestion, with more or less marked general atrophy, ranging from a little under weight to the extreme degree in which life is a constant burden.

4. The Cow's Milk Type.-While this group was the smallest in numbers, it was, nevertheless, distinct in its characteristics. The infants comprising this group were usually somewhat below the normal in weight, but in other respects they resembled breast-fed infants, although, with less adipose tissue, they seemed harder in flesh and more wiry; the color was always good; signs of rickets were inconspicuous; the teeth erupted normally; and the general resistance was good, as shown

* Read in the Section on Diseases of Children of the American Medical Association, at the Fifty-eighth Annual Session, held at Atlantlc City, June, 1907. 
by prompt recovery from whatever illness occasioned the risit. On questioning the mothers as to feeding. it was learned that cow's milk had been used with little, if any dilution-usually not more than a little limewater in each bottle. Such cases were numerous enough to impress on me the value of high proteid percentages, provided, of course, that the infant can digest them ; for it can not be too often repeated that each child must be studied separately, and not forced to take a certinin mixture simply because another child has thrived on it.

The term "low proteids" is relative and arbitrary, and as used in this title is held to be any percentage below half of that present in whole cow's milk. If we accept the figures of some chemists that human milk contains 1.5 per cent. proteid, and cow's milk 3.6 per cent., then a dilution of half the full strength of cow's milk will be seen to contain .3 per cent. more proteid than good human milk. It is an axiom in the artificial feeding of infants that cow's milk is harder to digest than human milk; it would, therefore, seem that per

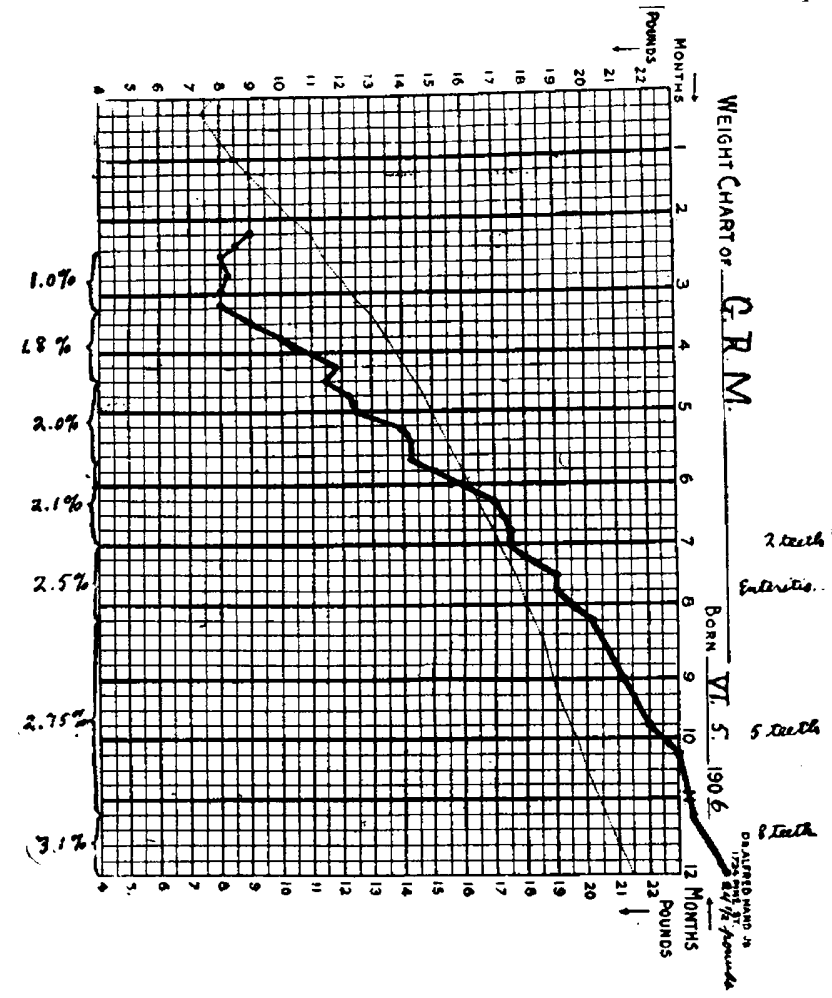

cent. for per cent. the former would yield less nourishment than the latter, and that consequently there is need of a higher percentage of proteid in artificial feeding than exists in human milk, in order that progress may be satistactory. Practically, this has been borne out in my own experience in watching effects on development of percentages above and below the half-and-half line. It may be offered in objection to this idea that as cow's milk is harder to digest than human milk, the percentage in artificial feeding must be either below, or not above, that of human milk, or else the infant's digestion will have a great strain thrown on it. It would reply to this that just as the individual is supposed to have more lung capacity than he needs for his usual demands, so it is conceivable that the digestive powers have some reserve, and would be able to handle a proteid of 1.8 per cent. in cow's milk feeding, although they were intended by Nature and had only been accustomed to handle 1.5 per cent. in human milk. I find this to be so for the great majority of healthy infants, who can start off any time after two or three montls of age on a mixture containing 1.8 per cent. proteid. There is another class comprising what have been called "difficult feeding cases," which is recruited largely from those who hare been bottle fed from birth, or who have had recent gastroenteritis, the members of which are unable to handle such a percentage unaided. If these infants are kept on low percentages which they seem able to digest, certain disadrantages will frequently result, and be manifest in the behavior or development of the children.

These disadvantages which appear in varying frequency are: Constipation; failure to gain steadily in weight, or else the gain is at a very slow rate; fretfulness and poor sleeping habits; the development of rachitis in greater or less degree; the frequent appearance of curds in the stools; anemia; an increase in the natural liability to develop acute enteritis, and in the susceptibility to various infections; and sometimes very markel infantile atrophy. It may be said in passing that a vicious circle is established often, the inability to hanlle the proteid clement and the general condition reacting on each other to the detriment of the infant.

There are two ways of handling such cases, quite different from each other; one may be called underfeeding; the other, for contrast, may be called sthenic feeding. The method of underfeeding aims to keep the proteid element at a low percentage, such as the digestive powers, which are below par, can handle by themselves, and percentages like the following are often given:

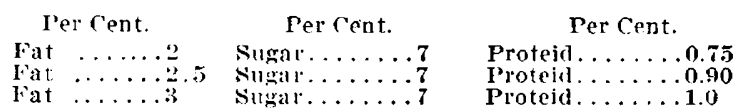

The aim, which is a laudable one, is to avoid overtaxing the system, and have it gradually develop the power to liandle stronger mixtures. We are sometimes compelled to resort to this method, but in my experience it is a slow, tedious process, and the upward climb is a hard one, and beset by the pitfalls mentioned above.

The method of sthenic feeding has as its aim so to modify the milk-mixtures that the infant will take those percentages which a normal infant of the corresponding. age takes. These percentages may be broadly stated, thus:

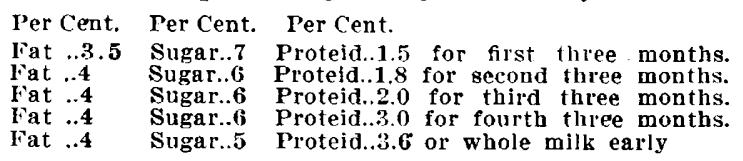

in the second year. In my own practice, both private and hospital, I get better results by this method than by tl:e former, although occasionally, much to my disappointment, I am compelled to give low proteid percentages.

It is but fitting to add that practically all of the infants whose feeding I direct receive clean milk, usually that receiving the certificates of the milk commission of the Philadelphia Pediatric Socicty, and that the mixtures are always made with a cereal decoction, such as barley-water. When it seems to be indicated, I do not hesitate to use predigested milk or to give either pepsin or pancreatin with the feeding, but it is never necessarv to use these aids for any great length of time or in manv cases. The average time is about two or three weeks. I have often been surprised to see infants whose digestive powers I had thought to be below par take and digest unaided, a stronger mixture when the former use of 1.5 per cent., 1.8 per cent. or 2 per cent. proteid harl bcen attended with colic, presence of curds in stools; and failure to gain in weight.

The sample weight-chart exhibited is self-explanatory. 


\section{DISCUSSION.}

Dr. Thomas S. Southworth, New York City, said that he has been in the habit of referring to a portion of this subject as the subnutrition which occurs in children during the latter half of the first year, while Dr. Hand brought out the subnutrition which may occur from low proteids in the early half of the first year. In the early teaching, he said, it was represented that if one could get the child up to a formula of F., 4; S., 7; P., 2, at six months the child would run along romfortably for several months with 2 per cent. proteids. This fallacy crops out all the time, although there is an unquestionable demand by a normal child for increasing proteid, in order to secure proper muscular development. Certainly, after the seventh month, if the child's digestion is normal, the proteids should often rise higher than 2 per cent. (estimating the proteids of whole milk at 4 per cent.). Another cause of proteid subnutrition arises frequently in summer. The children are doing well when they go out of town. The parents continue during the summer months with the same formula with which they left the eity, and there is timidity on the part of those who take up their feeding in the country in increasing the formula sufficiently. The result is they frequently come back below weight. It is possible in the summer to push up the proteids, even though the weather is warm. Dr. Southworth was glad to hear Dr. Hand say that in certain caseg curds, cr what are called curds, disappear with increased strength of the food. This, he said, requires careful discrimination to determine which cases may do that, but it certainly happens. He was glad also that Dr. Hand mentioned his use of a cereal drluent in seeding the higher proteids, because in discussing these matters it is necessary that one should understand just exactly what the other man does. The same results night not be obtained from an attempt to do it with a plain water diluent. A method often fails if the minor details are neglected.

Dr. Frank S. Churcinll, Chicago, expressed his surprise that Dr. Hand said nothing about the value of splitting the proteids. Of course, it is known that the proteid is a very complex substance, and that it varies in human milk and cow's milk. In human milk there is a large percentage of lact. albumin and a small percentage of caseinogen, whereas in cow's milk it is the reverse. This, he said, can be changed by feedling whey, and by using whey as a diluent in modified milk mixtures, feeding a baby proteid element in the shape of lact-albumin, which is easy of digestion, without increasing the more-difficult-to-digest caseinogen. If this were done it would not be necessary to use an aid to digestion. It is another step toward attempting to imitate the normal food of the baby.

Dr. R. B. GILBert, Louisville, referred to the use of milk from cows that are pregnant. Dairymen, he said, milk cows as long as the will give milk. The milk from a pregnant cow contains a large percentage of colostrum, and no matter how much pasteurization is given it, so long as that colostrum is in the milk, the baby is more or less sick. That is the difficulty of feeding a baby on one cow's milk. One-third of the cows in a dairy are pregnant, and one-third of the milk is affected. Dr. Gilbert urged that this always should be considered in the milk supply for the infant.

Dr. A. C. Mercen, Syracuse, N. Y., passed around a powder which represents whey, and which, he thought, is as nearly perfect as such a product can be. About one heaping teaspoonful of the powder to an ounce of water makes whey of the average formula.

DR. JoH. L. Morse, Boston, objected to the statement that a baby of a given age must have a certain percentage of proteid in its food. It is not the percentage of proteid, he said, that is of importance. but the total amount of proteid. This depends, moreover, more on the weight than on the age of the infant. A baby must have at least 2 grams of proteid per kilo in order to thrive and gain. It is not necessary to have more, although it is no disadvantage to have more, if the baby can digest it.

Dr. Charles Douglas, Detroit, considered the necessity for having enough proteid in the food to meet the necessities of growth as more important than the amount of fat. The chil dren who have been fed one, two or three months in an imperfect way, either as breast or hand-fed children, more frequently demonstrate injury to the fat than to the proteid digesting power. There is no such thing, he said, as establishing a rule for a certain amount of proteid at a given age. Weight is the one thing that gives anything like a fair guide to the amount of proteid which a child requires. Whether one is able to give just that amount or not is always a problem in rases of difficult feeding. It is important to know whether a child has normal digestive power. In many infants the proteids, he said, can be pushed beyond where Dr. Hand has placed them, and they will take care of it. The problem with some children is to hold it down. The majority of infants are held down either by their defective natural powers, or by their acquired indigestion. What has most strongly impressed Dr. Douglas is the general writing about the difficulties of proteid feeding. They are not so great as one would be led to believe, if the amount given each and every day is adjusted to the ability of the child to digest it properly. If the physician increases each food continuously, so long as the child is comfortable, and there is absence of gaseous discharges, bad odors and liquid stools, he will find himself able to raise each article in the child's dietary up to its digestive ability and always obtain the best result.

Dr. G. R. PrSek, New York, stated that, as a rule, the stool is examined at too respectful a distance. It may be reported as containing a great amount of curd or mucus. In fact, nurses innocently give incorrect reports of the character of the stools. Often the so-called curd is not found to be curd at all, but fat.

Dr. J. W. VAN Dersulce, Oak Park, Ill., has given higher proteids for the last three or four years than most physicians. He believes that one can feed high proteids with aid to the normal development, and that the child should be put early on 'whole mills; the nutrition will be better. It is essential to bear in mind the difference between therapeutic feeding and artificial feeding for a normal child. For a child fed on artificially prepared foods, the sooner it is placed on natural food, the better. He did not agree with Dr. Hand in holding off whole milk so long. In children under his care he has seldom found it necessary to postpone the use of whole milk after six months. This simply means that a normal child at six months of age can be given one or two feedings of pure cow's milk a day, if the breast is not giving enough. The addition of a little citrate of soda to the milk will be of advantage for the first few days. In the case of sick children, who have had one or more attacks of acute indigestion, he believes a point to be remembered is to feed in regard to the weight as against the age. A little, weazened-up, six-months-old baby that weighs a pound or two more than at birth, is not to be fed according to the amount given a baby of normal weight at six months. The musculature of the digestive tract is dilated, and this child should be fed with small amounts of high percentage food, rather than given a large amount of low percentage food at each feeding.

Dr. Alfred HANd, Philadelphia, agreed with Dr. Morse that one must not compel one child to take a mixture of certain percentages simply because another child had thrived on such an one, the moral being that each patient should be individualized; but nevertheless there must be some general plan of what a child at a certain age ought to take. Some times, Dr. Hand said, an infant can not tolerate high percentages. One striking instance of this case came under his observation some time ago. A prematurely born infant lost steadily in weight in spite of breast milk and modified cow's milk, in percentages ranging from 1 to 1.8. Peptonization was also used. At three and one-half months the child weighed four pounds and was finally in extremis, from which she was resuscitated by transfusion with normal saline solution. Then the use of whey was begun. In the next two months the child gained two pounds and seemed in a fair way to thrive. She was then taken to the country, where she succumbed to an enteritis. With regard to barley water, Dr. Hand's preference is for washed pearl barley, one tablespoonful to a pint-an easy proportion for the mother to remember-boiled until one-third has evaporated, 
when the water is strained off and used as a diluent. For convenience, he some times allows the use of barley flour, one teaspoonful to a pint, but he does not deem this to be quite so good. Colostrum in cow's milk, he said, is an important point. It remincled him of the plea by a prominent Philadelphia dairyman to reward industry and patronize the prosperous dairyman rather than, through sympathy, the one with only a few cows. According to the logic of chance, a child would be much more apt to get colostrum with every feeding from, say, a dairy of five cows with one pregnant and all of the mill mixed together before bottling, than from a dairy of 100 or more cows, where from 5 per cent. to 10 per cent. are pregnant at a time. In the large dairies the milk from 15 or 20 cows is put through the bottler at one time, and unless the pregnant cows are evenly distributed through the stalls, a child taking milk from this dairy would stand a good chance of getting colostrum milk only occasionally.

\section{PROPHYLAXIS IN EPIDEMIC CEREBRO- SPINAL MENINGITIS.*}

\section{A. SEIBERT, M.D.}

l'rofessor of Pediatrics, New York Polyclinic; Visiting Physician to St. Francis Hospital. NEW YORK CITY.

Epidemic cerebrospinal meningitis is a communicable discase. Its cause, the Meningococcus intracellularis, although first described by Weichselbaum twenty years agro, was not generally recognized as such until the bacteriologic work of recent epidemics had been reported on.

Taking the epidemic of 1905 in the province of Upper Silesia in Germany as an example, described by Westenhoeffer, ${ }^{1}$ we find that 3,102 persons were attacked and that 1,789 succumbed in this epidemic. During its course von Lingelaheim made over 3,000 tests at the bacteriologic station at Beuthen and found the meningococcus in the mucus taken from the nasopharynx in 93.8 per cent. of the patients.

This coincides with the finding of Councilman, Mallory and Wright in American epidemics. Ninety per cent. of the patients were children under 15 years of age.

In twenty-four healthy relatives of the patients Ostermann $^{2}$ found the meningococcus in the mucus taken from the nasopharynx seventeen times.

According to Kutscher ${ }^{3}$ the meningococci do not colonize in the nose but in the pharynx, preferably the nasopharynx. By introducing meningococci into the spinal canal of monkeys Flexner was able to produce inflammation of the meninges closely resembling cerebrospinal meningitis in man.

The biology of this organism is now well known. Outside of the human body it speedily suecumbs to sunlight and desiceation. It grows but scantily in artificial culture media at 98 degrees and not at all at 70 degrees Fahrenheit. According to Flexner it dies rapidly when suspended in salt solution.

Flatten, ${ }^{5}$ in his exhaustive report on the epidemic in the mining district of Oppeln in 1905, leaves no doubt that the meningococcus can survive in the warm moist atmosphere of the mines. We may thus summarize what we now know:

1. Epidemic cerebrospinal meningitis is communicable only by direct contact with fresh mucus from the nasopharynx of patients.

- Read in the Section on Diseases of Children of the American Medical Association, at the Fifty-eighth Annual Session, held at Atlantic City, June, 1907.

1. Berlin klto. Wochschr., No. 40, 1906

2. Deutseh med Wochschr. No 11, 1906

3. Rerlin klin. Wochschi. No 41, 1906 .

4. Journal of Exp. Medicine. March, 1907.

5. Hlatten: Klinlsches Yahrbuch, $x v, 1906$.
2. A person who has taken the germ from a patient may acquire meningitis.

3. Such a person may only acquire meningococcus pharyngitis and, thus acting as intermediary host, may carry this infection to others, near and far.

Kutscher, ${ }^{6}$ a member of the Institute for Infectious Discases in Berlin, in his article on "Practical Results Regarding the Etiology and Epidemiology of Cerebrospinal Meningitis," in closing his remarks, says: "It is necessary by all means to find a therapentic remedy which will kill the meningococcus in the nasopharyngeal mucus." (In parentheses I would like to add here: "not alone in the mucus but also in the mucosa.")

Both Kutscher and Westenhoeffer state that gargling, spraying and pharyngeal swabbing have so far not succecded in accomplishing this result. Jatile alone claims that he has succeeded in removing the meningococci from the nasopharynx by the use of pyocyanase, a product of the Bacillus pyocyaneus. He promises to publish his method later on.

That the usual methods of disinfection, without destroying the organisms in the upper pharynx of all persons exposed to contact infection, can not prevent the spread of this disease is the unanimous opinion of those best able to judge.

Postinasal catarrh is one of the most common ailments among children. 'The removal of adenoids and of enlarged tonsils alone does not cure the catarrh. To accomplish this the organisms inhabiting the affected parts must be killed. The removal of the mucus and even an effective surface disinfection will not suffice, because it does not reach the bacteria lodging deep in the mucosa. leep penetration of the disinfectant is, therefore, necessary to cure postnasal catarrh caused by meningococci or other bacteria.

During the last twelve years I have used a solution of equal parts of resorcin and alcohol to disinfect the nasopharynx. The alcohol must be heated before the resorcin is added. A plug of absorbent cotton wound around the end of an applicator, dipped into this solution, is introduced into the nasopharynx. Swabbing is not necessary. 'Two applications, one past each side of the uvula, are sufficient. The curved end of the applicator must be long enough to reach the ceiling of the nasopharinx. The cotton must be well soaked with the solution, but only so that drupping does not occur. The applicator must be bent so as to suit the length of the respective: nasopharynx. After two seconds the cotton may be withdrawn. The stomach must be empty. The contractions of the soft palate instantly press the fluid out of the cotton and into every fold of the mucous membrane. The alcohol penetrates deep into the mucosa with the resorein, and there destroys every organism it comes in contact with.

'These applications are best repeated every forty-eight hours. Six treatments usually suffice to stop all secretions of postnasal mucus in a chronic case. In recent cases two applications in one sitting usually suffice. Infants one month old bear this treatment as well, and often better, than adults. Hundreds of cases treated solely in this manner have always given the desired result. The first application is usually felt during the next twenty-four hours, the later ones cause no inconvenience. The two cardinal symptoms of postnasal catarrh, namely, secretion of mucus and multiple swelling of the posterior cervical lymphnodes, usually disappear after three to six applications.

6. Deutsch. med. Wochschr., No. 46, 1906

7. Milnch, med. Wochschi., No, 29, 1906. 